\title{
Towards a Framework for Sustainable Knowledge Management in Organisations in Developing Countries
}

\author{
Adekunle Okunoye \\ Turku Center for Computer Science \\ University of Turku/Department of Information Technology \\ adeokun@cs.utu.fi
}

\begin{abstract}
Several models and frameworks abound to guide knowledge management (KM) implementation and strategy in organisations. These are mostly based on experiences and studies in Western industrialised countries that are already becoming knowledge economies. Application of these models and frameworks in developing countries might not yield expected results. For organisations in developing countries to participate in this new economy, there is need to understand the issues of KM in their local context. Towards this goal, we present the results of an empirical study of KM in six research organisations in sub-Saharan Africa. Requirements for a context specific framework for KM in organisations in developing countries are then presented.
\end{abstract}

Keywords: Knowledge management, research organisations, information technology infrastructure, developing countries, sub-Saharan Africa

\section{INTRODUCTION}

Knowledge has been observed to have an important role among the traditional resources of land, labour, and capital in creating and sustaining economic value. There is a growing demand for knowledge-based products and services. This is changing the structure of the global economy. The role of knowledge in achieving competitive advantage becomes an important management issue in all sectors [14][37]. As a result, many organisations are exploring the emerging field of knowledge management (KM). Knowledge

The original version of this chapter was revised: The copyright line was incorrect. This has been corrected. The Erratum to this chapter is available at DOI: 10.1007/978-0-387-35609-9_29 
organisations like research organisations in sub-Saharan Africa also need effective $\mathrm{KM}$ to be productive, rèsponsive, competent, and innovative in order to win highly competitive grants to support their research and to produce knowledge essential for development. They are under a growing pressure on quality assurance and cost reduction which require finding a way of improving their knowledge activities.

The American Productivity and Quality Center [3] has defined KM to be a conscious strategy of getting the right knowledge to the right people at the right time and of helping people to share and put information into action in ways that strive to improve organizational performance. $\mathrm{KM}$ is a complex process that must be supported by a strong foundation of enablers. The enablers for KM include strategy and leadership, culture, measurement, and technology. Each of these must be designed and managed in alignment with the other and in support of the process. To capitalise on knowledge, an organisation must be swift in balancing its KM activities, which require changes in socio-cultural and organisational processes, technologies and techniques [6].

A more holistic view of KM requires interplay between the socio-cultural and organisational components and technological components [2][35], which highlight the interweaving of social and technical factors in the way people work. However, most of the current KM models are biased towards the Western organisational environment from where they had the inputs for their design and where they have been tested and applied. There has been also much focus on the technological infrastructure in sub-Saharan Africa, both at micro and macro levels [13][26][28][29] and there is some understanding of how this affects organisational and national development. To design effective, sustainable KM, relevant to organisations in sub-Saharan Africa, there is need to further understand the pertaining socio-cultural and organisational issues.

We designed a field study [32] and gathered data on KM in six research organisations in Nigeria and The Gambia during winter 2001. Nigeria accounts for a sixth of the entire population of African countries and thus it is representative of the socio-cultural issues common in the region. The Gambia is one of the smallest countries and it provides a good comparison to Nigeria. The aim of the research is to have a detailed understanding of KM processes and the enablers in organisations in developing countries for developing a model that is specific to their situation.

The analysis of the data indicates that many of the problems encountered are not limited to technology alone but they relate also to socio-cultural and organisational issues. These can include managerial issues, dependency on key personnel, tight schedules, short-term planning due to the project culture, persistent under-funding, long delays in communications, and so 
forth. Based on this insight, we work towards a framework that could later be developed and assessed as a model for KM that would incorporate each of these concerns and the local factors specific to developing countries, to guide its implementation.

\section{THE NEED FOR A CONTEXT SPECIFIC MODEL}

The diffusion and effective utilisation of information technologies (IT) that could support KM have not spread evenly over the world [26]. Information technology is more utilised in western industrialised nations and less in the developing nations. This is due to several factors, including availability of funds and adequate expertise to manage the technologies. According to Arunachalam [4], most organisations in developing countries, especially those with large populations (such as Nigeria in our study), do not have the necessary infrastructure to contribute as equal partners in the worldwide enterprise of knowledge production and dissemination. In addition, in several of the information technology installations that were adapted for organisations in developing countries, local factors were not taken into account. This has resulted in outcomes that do not fit the needs of the direct beneficiaries in the developing nations [12].

Most efforts in managing knowledge in sub-Saharan Africa have been at the country and community level. The key message of the World Development Report for Africa [40] is that most countries on the continent need to do much more and faster to increase their knowledge base, to invest in educating their people, and to take advantage of the new technologies for acquiring and disseminating knowledge. Countries that postpone this will fall behind those that move faster. Undesirable development can be hard to remedy.

For developing nations to compete and profit in the global marketplace of today, there needs to be efficient ways to leverage existing knowledge. The development agencies efforts were reflected in the World Bank Report of 1998 that was specifically devoted to knowledge for development. The report examines the role of knowledge in advancing economic and social well-being. Because knowledge is at the heart of economic growth and sustainable development, understanding how people and societies acquire and use it-and why they sometimes fail to do so-is essential to improving peoples' lives, especially the lives of the poor.

The report suggests three lessons that are particularly important to the welfare of the people in developing countries. First, developing countries must institute policies that enable them to narrow the knowledge gaps separating poor countries from rich. Second, developing country 
governments, multilateral institutions, non-governmental organizations, and the private sector must work together-to strengthen the institutions needed to address the information problems that cause markets and governments to fail. Third, no matter how effective these endeavours are, problems with knowledge will persist. But recognizing that knowledge is at the core of all our development efforts will allow us to discover unexpected solutions to seemingly intractable problems.

While all these seem to be relevant to the national governments, we should remember that organisational development also has a great influence on national development. According to Davenport and Grover [15], KM is no longer in the domain of organisations that are primarily concerned with selling knowledge-based products but it is an integral business function for many organisations that want to remain competitive based on the effective management of their intellectual resources.

For these reasons, organisations in developing nations should also begin to consider KM. Unfortunately, the only available models and examples are based on organisations in Western industrialised countries. The problems associated with applying these in developing countries could be related to the problems of IT transfer to developing countries. According to Straub, Loch and Hill [39]

"The transfer of information technology (model) from industrialised to developing countries generally involves a process of injecting the technology (model) of the industrialised world and its associated methodologies into a developing nation host. In that technology (model), designed and produced in developed countries, is likely culturally-biased in favour of industrialised socio-cultural systems, technology (model) transferred to developing countries meets cultural resistance." [39], p.6

In the case of $\mathrm{KM}$, it is not only limited to cultural-based resistance but other variables might not appropriate in the local settings. When this happens, the model is usually not sustainable-if at all usable-in the first instance [25][26][28]. The adoption and use of such a technology (model) varies according to local socio-cultural and organisational contexts [39]. The recent study conducted by Becerra-Fernandez and Sabherwal [5] also suggests that KM should be implemented with due consideration to the particular context of each organisation. For these reasons we studied the local socio-cultural, organisational, technological, and operating environmental context and use our results for constructing the new framework. 
There have been specific KM models for organisations in the West and Japan in the early 1990s [18]. To avoid the mistakes associated with the technology transfer, where not meeting the recipient nations' needs is a common problem, a context specific model would be appropriate. Although the circumstances in developing countries call for a model in their own specific context, the contextual issues in $\mathrm{KM}$ are also of interest globally, and not only in developing countries.

\section{EXISTING FRAMEWORKS AS A STARTING POINT}

Organisations have been viewed as complex systems in which at least four sets of variables interact. These are, as identified by Leavitt [22]: task variables, structural variables, technological variables, and human variables. Each of these sets of variables could bring about changes in organisations. Taken together, we could call the task, structural, and human variables the social variables. Leavitt [22] suggested a balance between these social and technical variables to effect changes in organisation.

This sociotechnical approach of Leavitt has been widely adopted and cited [17][27] as a basis for understanding organisational changes. Pan and Scarbrough [36][35] have specifically used this approach in exploring KM in organisations. They used the sociotechnical perspective to trace the interaction between $\mathrm{KM}$ practices in organisational context at Buckman Laboratories. They suggested interplay between KM system as the technical context and the organisational issues like management and leadership as the social context. Sena and Shani [38] have also used sociotechnical approach in proposing an alternative framework for intellectual capital and knowledge creation.

About the same time Leavitt proposed the Diamond, Trist [42][35][41] suggested the term sociotechnical to describe a method of viewing organisations. This method emphasises the interrelatedness of the functioning of the socio-cultural, organisational and technological subsystems of the organisation, and the relation of the organisation as a whole to the environment in which it operates. Most of the recent frameworks proposed for KM also comprise of sociotechnical components (e.g. [2][3]). Exclusive focus on the social or the technical components may effect a change but does not enable a sustainable competitive advantage, one of the primary aims of KM [6][11]. Since our aim is to develop a sustainable KM model that considers interaction between all the organisation's social and technical subsystems, we found the sociotechnical approach relevant and it thus prompts our interest in studying each of the subsystems in detail. 
The technical aspect has been taken for granted in the Western industrialised countries [20]. Even though most of the KM models and frameworks include a technological component, the implementation usually focuses more on other enablers. We are not advocating for a technologically focused model of KM but a model that would give due consideration to both social and technical components, as these become a reality in organisations.

We have used an existing model of KM that was developed by the American Productivity and Quality Center (APQC). This model considers $\mathrm{KM}$ a complex process that must be supported by a strong foundation of enablers. The enablers that the APQC model includes are strategy and leadership, culture, measurement, and technology. The process usually involves several of the following stages or sub-processes in the use of knowledge: create, identify, collect, organize, share, adapt, and use. We used the instrument designed by Bukowitz and Williams [9] to assess each of the processes. We also sought qualitative data to assess the enablers. A list of IT Infrastructure, developed and tested by Broadbent, Weills, and St. Clair [7][8], was used to supplement the data on technology. While we were trying to assess the current state of $\mathrm{KM}$ in these organisations, we were also investigating the appropriateness of the earlier findings and sought any new phenomena that could be a KM enabler in our case organisations.

\section{THE RESULTS OF THE EMPIRICAL STUDY}

A multiple case study of six research organisations in sub-Saharan Africa was conducted. Research scientists, librarians, IT managers and administrative staffs were interviewed and observed for a period of about two weeks in each organisation. This is in addition to the use of questionnaires and analysis of historical documents. The research methods are reported in detail in Okunoye and Karsten [32] and results about the enabling role of technology in KM in Okunoye and Karsten [34]. The detailed results of the study have been reported in Okunoye [30]. For the purpose of this paper we will summarise the key findings, which could explain the source of key components of our suggested model.

The KM diagnostic revealed that the research organisations generally performed well in their efforts in creating, finding and collecting internal knowledge and best practices. They averaged in sharing and understanding those practises and were weak in adapting and applying the practises to new situations. They all have a well-established process of getting knowledge, which does not necessarily involve much use of information technology. Most of the organisations still use manual ways of managing documents through filing. Most of the organisations have been able to incorporate 
learning into their normal work process. They all support ways of learning from experience at the completion of a research project through seminars and workshops where the process is reviewed.

Much effort is expended on proper documentation, but the learning process is still affected by inadequacies in this. Also proper succession plans may be lacking. Information technologies could well be used to make these easier to manage. The informants all recognise the importance of knowledge repositories through which researchers can share and acquire knowledge but these developments are being affected by lack of trust among the researchers in some of the organisations: they believe that their knowledge is their source of power. Sparing time to contribute to the repositories is another main constrain.

Only one of the organisations has a KM initiative. Most of the others have, however, separate strategies to improve communication and learning, which have been found to facilitate also KM. They use seminars and workshops for learning and for knowledge sharing. They all use various forms of training (on-the-job, online, distance training) to develop the skills and expertise of their staff. All the organisations are structured in a matrix form, which supports a knowledge sharing culture. They all operate in collegiate ways and they use multidisciplinary project arrangements to encourage cooperation among the researchers.

Out of the twenty-three items assessed on the IT infrastructure services list [7], only data management that is common to all the organisations. Three of the organisations had more than twelve IT infrastructure services while the remaining three had less that ten [34]. Although they all expressed the importance of having a local area network, it was fully available only in two of the organisations. These organizations also happened to have the highest number of IT infrastructure services. Assessment of other technologies also revealed the Internet as common to all the organisations with variations in the level of other infrastructures [31][33]. The researchers see the Internet important in gaining quick access to funding resources required to support new ideas and to enable innovation, and more importantly, to access the collective knowledge of their colleagues.

The leaders of the organisations encourage communication and collaboration. They recognise and reward good ideas and innovations. They use face-to-face periodic regular meetings for deliberation, planning and decision-making. The organisational culture and structures of the organisations provide a good ground for KM. Most of the research programmes in the organisations are multidisciplinary and interdisciplinary in nature, making everybody useful and important to each other and hence supportive of knowledge sharing. There are forums for informal networking 
of the experts. The issues of trust and how to reward knowledge sharing are among the concerns expressed in the organisations.

An effective KM is expected to bring some benefits and changes to the organisations. Most of the interviewees believe that applications of appropriate IT will shorten their response times to key issues by giving them access to the information they need when they need it. This could indirectly improve productivity. While the IT capability varies in the organisations, they all agreed that using technology effectively would allow them to capture and share best practices and other explicit knowledge assets. This might also shorten cycle times and minimize duplication of their efforts.

Due to the location of the organisations, they all recognise the advantages of online training and distance learning that the technology has made available. However, they still have problems with the technology that need to be solved before benefiting fully from it. These problems include connectivity and access, improvements of the skills and better expertise acquisition which could translate to more competence and efficiency on the job.

We found one form of enablers that is not often talked about in the previous KM models and frameworks (with the exceptions of [10] and [19]), but which becomes essential for the success of KM in these settings. These are usually external factors that we refer to as the operating environment influence, which cannot be directly controlled by the organisation. The operating environment (the funding level, governmental commitment, other infrastructures like transport, telecommunication, electricity etc.) varies from organisation to organisation, between countries, and also from one site to another in a country.

During the interviews with the scientists in all national organisations in both countries, they expressed the problem of low funding and how it is affecting their efforts in getting the required knowledge to do their work and hence their productivity. Apart from spending their own resources to acquire technology required for their job, the low funding does not allow the organisation to invest in the right kind of IT infrastructure, which they all agree could facilitate their communication and knowledge sharing. However, there is difference in international organisations where they have considerably higher funding. For example, the IT infrastructure in international organisations is better than in the national organisations [31]. However, even sufficient funding cannot always overcome the problems in the surrounding society. 


\section{TOWARDS SUSTAINABLE KNOWLEDGE MANAGEMENT}

Like most of the KM initiatives in the West [1][9][14][16][23], the efforts in our case organisations are also geared towards managing the processes and providing an enabling environment-although these may not be mentioned explicitly. As described above, all the enablers included in the current models and frameworks [19][21] are also essential for organisations in developing countries.

One key difference we found was that the case organisations in the West understand the issue of technology use better than in developing countries and therefore they can concentrate more on other enablers such as culture, structure and other organisational issues. That is, while the current models are tilted towards the socio-cultural and organisational enablers, the unavailability of technology in expected proportion in developing countries requires its careful and detailed inclusion in the model. While it is not possible for each organisation to have the same level of IT infrastructure, they should still be able to apply the model as adjusted to their circumstances. Therefore, an explicit definition of IT should be included.

Another issue that calls for serious consideration are the operating environment influences. These influences operate from several directions. The differences in the level of availability of national information infrastructure, which usually affects organisational infrastructure, emphasise again the detailed inclusion of the technology component in the KM model for developing nations.

Based on our findings in these organisations, we will outline our suggestions for a context specific framework by using the Leavitt Diamond as a starting point. The Diamond needs to be adapted to guide KM in developing countries. First, we add a component to represent the sociocultural elements (see Figure 1). For example, the age stratification and accompanying respect in traditional African societies automatically arrogates some power to the elders. It does not allow juniors to question the authority or views of seniors. This culture may then be filtered into organisational settings where supervisors could assume the role of the elders. Thus could then influence the way the subordinates integrate the knowledge from their sources.

While each of the other components could be specific to each organisation, the contextual issues are more pronounced in the socio-cultural aspect. In the context of the national organisations, where most of the staffs are national, the above could be noticed easily. It might be less pronounced in the international organisations, where the members of staff are composed of various nationals. Application of the same model in these organisations 
might be inefficient, even when the organisations were are located in the same country.

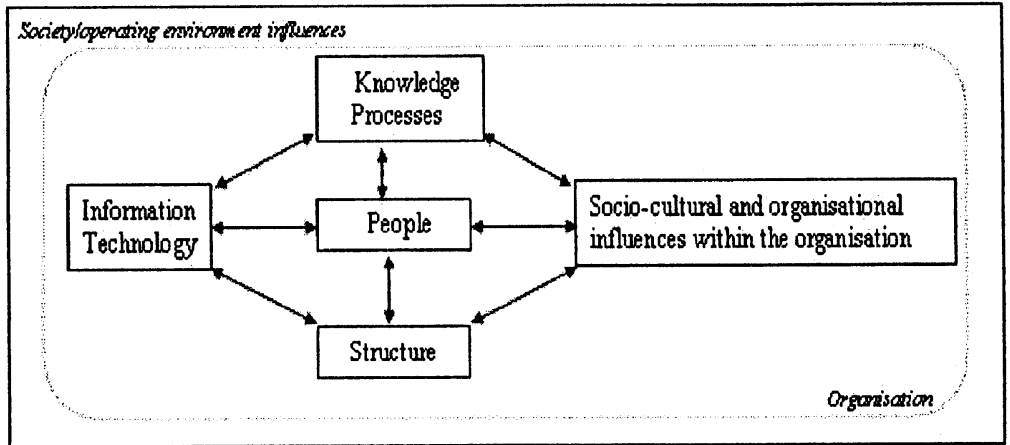

Figure 1: The suggested framework for sustainable knowledge management

To further expand on the Diamond, the knowledge tasks ("processes") could be seen to require a better organisation than currently. They then would need to be supported by an organisational structure that puts more emphasis on the kind of coordination and supervision which would enable empowerment, that is, power and control to be more distributed among the staff. This would enable decreasing supervision and encouraging independent work teams which focus collectively on the goals of the organisation and perceive the achievement as team-based while acknowledging individual contributions. As also suggested by Leavitt [22], the knowledge processes have to be streamlined with the available information technology infrastructure. However, the people are the connecting link to all these components. Together, as suggested also by Bhatt [6], the dimensions have to be balanced. Moreover, all this has to be aligned with the goal and the vision of the organisation while taking into consideration the society in which they operate.

Thus, our proposed framework for KM in organisations in developing countries should consider the socio-cultural and organisational factors, technological factors and operating environmental factors, based on what we found in our study and existing literatures. All these should be aligned with the aim of each organisation. The proposed model should also consider each knowledge process and provide adequate enablers to support each of these. The technological component should be explicit on the degree of availability of IT and how the business goals increase with increase in the application and availability of technology. The business goals have been summarised broadly as responsiveness, innovation, competency and efficiency [24]. These were confirmed as the expectations also in our case organisations. The processes that should be supported could also be broadly summarised as 
knowledge generation and adaptation, knowledge codification, refinement, storage and organisation, knowledge transmission, distribution or deployment, and knowledge application or leveraging.

The major problem in representing the goals and subsequently measuring them in our proposed framework is in the dynamism of the associated variables. We cannot predict the scope of any of the goals even with proper application of the framework. Thus, the application and determination of its success will vary from one organisation to another and their subjective interpretations. In a similar manner, we would like to show how each of the processes could improve with adjustment or balancing of the enablers, but it would only add to the complexity of the framework without adding the contextual point we propose. Nevertheless, despite all these limitations, it is still essential to try out the realities and the applicability of this framework in practice. Only in this way the problems can be clarified and a path to a more refined model be paved.

\section{REFERENCES}

[1] Alavi M. and Harley, B. (1999). Knowledge Management Systems: Implications and Opportunities for Data Warehousing. Journal of Data Warehousing Vol. 4 Issue $1 \mathrm{pp}$. 2-7.

[2] Alavi M., Knowledge Management and Knowledge Management System in Proceedings of $18^{\text {th }}$ International Conference on Information Systems, Atlanta Georgia December 14-17, 1997

[3] American Productivity and Quality Center (APQC), Knowledge Management: Consortium Benchmarking Study Final Report 1996 Available in http://www.store.apqc.org/reports/Summary/know-mng.pdf [Accessed April 3rd, 2000]

[4] Arunachalam, S., Information Technology: What does it mean for scientists and scholars in the developing world. Bulletin of the American Society for Information Sciences, 1999 Vol. 25 pp 21-25

[5] Becerra-Fernandez I. and Sabherwal R. Organisational Knowledge Management: A Contigency Perspective, Journal of Management Information Systems Summer 2001 Vol 18 No 1 pp23-55

[6] Bhatt G., Knowledge Management in Organisations: Examining the interaction between technologies, techniques, and people. Journal of Knowledge Management, Vol. 5 No. 1 2001

[7] Broadbent M. and Weill P., Management by Maxim: How business and IT managers can create IT Infrastructures, Sloan Management Review, Spring 1997

[8] Broadbent M., Weill P., St. Clair D., The Implications of Information Technology Infrastructure for Business Process Redesign, MIS Quarterly 1999 Vol 23 pp 159-182

[9] Bukowitz W. R., Williams R. L. The Knowledge Management Fieldbook, Pearson Education Limited: London, 1999.

[10] Calaberese F. A Suggested Framework of Key Elements Defining Effective Enterprise Knowledge Management Programs. George Washington University, The School of Engineering and Applied Science, PhD Dissertation, May 2000 
[11] CIO, Knowledge Management: Collaborating for a Competitive Edge. White Paper Library, 2000 Available at http://www.cio.com/sponsors $/ 0600 \mathrm{~km} / \mathrm{index}$.html [Accessed 26th July 2001]

[12] Cyamukungu, M., Development strategies for an African computer network. Information Technology for Development; Oct 1996 Vol 7 pp 91-94

[13] Dasgupta S., Agarwal D., Ioannidis A., Determinants of information technology adoption: An extension of existing models to firms in a developing country. Journal of Global Information Management, Jul-Sep 1999 Vol. 7 pp 30-40

[14] Davenport T. and Prusak, L. Working Knowledge: How organizations manage what they know. Harvard Business School Press: Cambridge, MA, 1998.

[15] Davenport, T. and Grover. V. General Perspectives on Knowledge Management: Fostering a Research Agenda. Journal of Management Information Systems $2001 \mathrm{Vol}$ 18 , No 1 pp3-4

[16] Earl M. Knowledge Management Strategies: Toward a Taxonomy. Journal of Management Information Systems Summer 2001 Vol. 18 No 1 pp. 215-233

[17] El Sawy O., Redesigning Enterprise Processes for e-Business. McGraw-Hill, 2000

[18] Hedlund G. and Nonaka I., Models of Knowledge Management in the West and Japan, in Lorange et al. (eds.), Implementing Strategic Process: Change, Learning and Cooperation, London Basil Blackwell, 1993

[19] Holsapple C. and Joshi K. An Investigation of Factors That Influence The Management of Knowledge in Organisations. Journal of Strategic Information Systems 2000, Vol. 9 , Issue 2-3, pp.235-261.

[20] Holtshouse D. Foreward in Borghoff, U. and Pareschi, R., Eds. Information Technology for Knowledge Management. 1998 Berlin, Springer-Verlag.

[21] Lai H. and Chu T., Knowledge Management: A Theoretical Frameworks and Industrial Cases, Proceedings of the $33^{\text {rd }}$ Hawaii International Conference on System Sciences 2000

[22] Leavitt H.J., Applied organisational change in industry: Structural, technological, and humanistic approaches, in March J. (ed), Handbook of Organisations, Rand McNally \& Co. Chicago 1965, pp.1144-1170

[23] Leonard-Barton D., Wellsprings of Knowledge: Building and Sustaining the Sources of Innovation, Harvard Business School Press: Cambridge, MA,, May 1998

[24] Lotus Development Corporation, White Paper on Lotus, IBM, and Knowledge Management. January 1998

[25] Morales-Gomez, D. and Melesse, M. Utilising Information and Communication Technologies for Development: The Social Dimensions. Information Technology for Development, Vol. 8 Issue 1, 1998, pp. 3-14.

[26] Moyo, L. M., Information technology strategies for Africa's survival in the twenty-first century: IT all pervasive. Information Technology for Development; Mar 1996 Vol 7 pp17-

[27] Mumford E, Designing Human Systems for Health Care: The ETHICS Method, Eight Associates, Cheshire, 1993

[28] Odedra M., Lawrie M., Bennett M., and Goodman S., International Perspectives: SubSaharan Africa: A Technological Desert, Communications of the ACM, Feb. 1993.

[29] Ojo S. O., Socio-Cultural and Organisational Issues in IT Applications in Nigeria, In: Bhatnagar, S. C. \& Odedra, M. (Eds.): Social Implications of Computers in Developing Countries, New Delhi: Tate McGraw-Hill, 1992, pp 189-199

[30] Okunoye A., Information Technology Infrastructure and Knowledge Management in sub-Saharan Africa: An Empirical Investigation. University of Jyvaskyla, Department of Computer Science and Information Systems, MSc. thesis 2001 
[31] Okunoye A. North in the South: Comparative Analysis of Information Technology Infrastructure in National versus International Research Organizations in Sub-Saharan Africa. In Proceedings of Third Annual Global Information Technology Management (GITM) World Conference, June, 2002 in New York, USA

[32] Okunoye, A, and Karsten, H. Information Technology Infrastructure and Knowledge Management in sub-Saharan Africa: Research in Progress. In Proceedings of Second Annual Global Information Technology Management (GITM) World Conference, June 10-12, 2001 in Dallas, TX, USA

[33] Okunoye, A, and Karsten, H. Now and Then: Internet Use in Research in sub-Saharan Africa. IFIP WG 9.4 Conference, May 2002, Bangalore India

[34] Okunoye, A, and Karsten, H. ITI as enabler of knowledge management: empirical perspective from research organisations in sub-Saharan Africa. HICSS-35 Cconference, January 2002, Hawaii

[35] Pan S. and Scarbrough H., A Socio-Technical View of Knowledge Sharing at Buckman Laboratories. Journal of Knowledge Management. Vol. 2 No. 11998

[36] Pan S. and Scarbrough H., Knowledge Management in Practice: An Exploratory Case Study. Technology Analysis and Strategic Management; Vol. 11 Issue 31999

[37] Sammons P., Smart Things to Know about Knowledge Management (Review), Supply Management, Feb 10, 2000 pp 42

[38] Sena J. and Shani A. Intellectual Capital and Knowledge Creation: Towards an Alternative Framework In Liebowitz J. (Ed.) Knowledge Management Handbook. Boca Raton: CRC Press, 1999

[39] Straub D., Loch K., and Hill C. "Transfer of Information Technology to Developing Countries: A Test of Cultural Influence Modelling in the Arab World" Journal of Global Information Management, 9, 4 (2001), 6-28

[40] The World Bank Group, World Bank Report 1998/99: Knowledge for Development Available from http://www.worldbank.org/ks/html/pubs_pres.html 1999 [Accessed April $3^{\text {rd } 2000]}$

[41] Trist E. The Socio-Technical Perspective. The Evolution of Sociotechnical Systems as Conceptual Framework and as an Action Research Program. In Van de Ven A. and Joyce W. (eds.) Perspectives on Organisation Design and Behaviour. 1981 Wiley, pp. 49-75

[42] Trist, E., Higgin, G., Murray, H., \& Pollock, A. Organizational Choice. London, UK: Tavistock. 1963 